\title{
Ignorantus, ignoranta, ignorantum [1]
}

Xavier Martin

PD Dr méd., membre de la FMH

Cet article rappelle à la fois les causes de l'augmentation des primes d'assurance maladie et la politique qui conduit à ce qu'elles croissent inlassablement ainsi que les conséquences des décisions prises sur l'évolution inquiétante des coûts des arrêts de travail. Enfin, les voies à suivre pour une gestion efficace du système de santé sont proposées.

L'auteur de ces lignes a été observateur des mutations survenues dans le système de santé dès 1971 et jusqu'à ce jour. D'abord comme étudiant et rédacteur du Journal des étudiants en médecine de l'Université de Lausanne et plus récemment comme président de l'une de nos sociétés médicales nationales.

Depuis 47 ans que j'observe la gestion de la santé en Suisse, je suis consterné par l'ignorance réelle ou feinte des responsables de la Santé publique.

Pour résoudre un problème, il faut se poser les bonnes questions et rassembler les données disponibles.

\section{La première question est: qu'est-ce qui coûte le plus cher, la maladie ou ses conséquences?}

Ce sont ses conséquences, en effet, une épidémie de grippe par exemple qui coûte 100 millions de francs en soins médicaux et qui est responsable d'environ 5 jours d'arrêts de travail par malade coûte 200 millions de francs en arrêts de travail [2]. Si l'on prend les accidents du travail ou les accidents de la circulation c'est pire [3] et c'est jusqu'à 5 qu'il faut multiplier les frais médicaux engendrés par les arrêts de travail.

\section{Zusammenfassung}

Dieser Artikel befasst sich zum einen mit den Ursachen für die Prämienerhöhung bei den Krankenversicherungen und mit der Politik, die zu immer weiteren Erhöhungen führt, und zum anderen mit den Konsequenzen der entsprechenden Entscheidungnahmen für die beunruhigende Kostenentwicklung aus den Krankmeldungen. Daran anschliessend werden Mittel und Wege für ein effizientes Management des Gesundheitssystems aufgezeigt.
Cependant, les arrêts de travail les plus coûteux ne sont pas ceux qui viennent d'être mentionnés, mais ceux engendrés par les listes d'attente. Si un chauffeur de poids-lourd doit attendre trois mois pour être opéré de la cataracte ou d'un tunnel carpien par exemple, cela représentera 90 jours d'arrêts de travail qui coûteront plus de 10 fois le coût des frais médicaux.

Si les coûts des arrêts de travail ne coûtaient que le triple des coûts des frais médicaux, cela présenterait déjà 240 milliards de francs par an.

\section{La deuxième question est dès lors évi- dente: que doit-on faire pour diminuer la durée des arrêts de travail?}

Pour diminuer la durée des arrêts de travail, il faut supprimer les listes d'attente et surtout empêcher qu'elles ne se forment. Dans ce but il est donc nécessaire que la prise en charge soit rapide et efficace et pour ce faire que le nombre de médecins soit optimal et que leur rémunération soit adéquate.

Or, depuis l'introduction du TARMED en 2003 et plus encore depuis les modifications de 2018, la rémunération du médecin pour la plupart des interventions ne respecte pas l'art. 43 alinéa 4 de la LAMal soit que les tarifs et les prix doivent être fixés d'après les règles applicables en économie d'entreprise.

Ces baisses de tarifs ont été décidées par des gens qui mélangent chiffres d'affaire des cabinets médicaux et salaires des médecins, assurances de base (LAMal) et assurances complémentaires (LCA) ainsi que les honoraires privés à la charge ni de la LAMal ni de la LCA. Tant d'incompétence à la tête de l'Etat est hallucinant. Les conséquences de ces décisions aberrantes sont claires. En effet, elles vont provoquer le transfert de la 
plupart des interventions courantes du privé au public. Or, la capacité des institutions subventionnées par l'Etat est insuffisante et provoquera inévitablement la formation de listes d'attente et l'explosion des coûts des arrêts de travail.

Le durcissement actuel de la clause $\mathrm{du}$ besoin va également favoriser la formation de listes d'attente, puisqu'il va diminuer le nombre de médecins disponible pour une population vieillissante qui a besoin de davantage de soins.

Pour diminuer les coûts, il faut donc faire l'inverse de ce qui a été fait jusqu'alors.

\section{Si ce qui coûte le plus cher sont les arrêts de travail, qu'en est-il du coût des soins médicaux et des primes de caisses maladie?}

Les causes de l'augmentation des primes des caisses maladie sont bien connues et sont essentiellement liées à trois choses, le transfert des opérations et autres gestes invasifs de l'hospitalisation à l'ambulatoire, les progrès de la médecine et le vieillissement de la population. La seule intervention efficace à long terme, et sur laquelle l'on peut agir, est le transfert des opérations et des autres gestes invasifs de l'hospitalisation à l'ambulatoire, puisque nous n'avons aucune influence directe sur le vieillissement de la population et les progrès de la médecine. Pour rappel, je cite: «Le secteur ambulatoire est financé à 100\% par les primes, alors que le secteur hospitalier est en grande partie financé par l'impôt cantonal» [4].

\section{Cependant cette augmentation de l'impôt sera sociale puisqu'elle sera payée comme tout impôt au prorata du revenu.}

Dès lors, il faut que les opérations et autres gestes invasifs, pratiqués en ambulatoire ou en hospitalisation, soient pris en charge intégralement par les cantons, comme cela était le cas lorsque l'ambulatoire chirurgical ou interventionnel était l'exception.

La prise en charge de toutes interventions ambulatoires ou en hospitalisation par les cantons va forcément entraîner une augmentation des impôts. Cependant cette augmentation de l'impôt sera sociale puisqu'elle sera payée comme tout impôt au prorata du revenu. Cette hausse de l'impôt sera compensée par une réduction des primes d'assurance maladie qui profitera aux revenus les plus faibles.
La mise en place de ce système est adéquate puisque les structures nécessaires existent.

En effet, la facturation des intervenions ambulatoires continuera à être traitée par les caisses maladie qui les contrôleront, comme cela se fait déjà aujourd'hui, et au lieu de les payer, elles les transmettront aux cantons qui les payeront.

Enfin pour éviter une médecine à plusieurs vitesses, les franchises seront supprimées pour les interventions chirurgicales ou invasives et pondérées pour l'ambulatoire non chirurgical et non invasif.

Cette correction indispensable est urgente. En effet, les thérapies géniques sont devenues réalité et le coût de tels traitements se compte en millions de francs et ces coûts énormes vont venir s'ajouter au flux continu du transfert de l'hospitalisation vers l'ambulatoire.

Qu'une caissière de supermarché paye la même prime d'assurance de base qu'un conseiller d'Etat ou qu'un conseiller fédéral est asocial, indécent [5] et honteux.

\section{Et le salaire des médecins, participe-t-il à l'augmentation des coûts?}

Là encore, il suffit de rassembler les données à notre disposition, soit les statistiques de l'Office fédéral de la statistique et les publications annuelles depuis 1981 d'Interpharm, entre autres.

Selon ces sources, le salaire des médecins baisse depuis 1982.

Pourquoi dès lors s'attaquer aux salaires des médecins?

A qui profite cette désinformation? Je vous laisse juge. Nos autorités réclamant de la transparence à propos du salaire des médecins ont été servies par la publication du communiqué de presse du 30 janvier 2018 de 
la Société vaudoise de médecine [6] qui dispose d'un centre de confiance par lequel transite l'essentiel des factures TARMED des médecins vaudois. Manque de chance pour ces mêmes autorités, le revenu moyen des médecins vaudois installés est l'équivalent d'un salaire annuel de 120000 CHF sur l'ensemble d'une carrière.

Lorsque l'on sait que les médecins installés travaillent en moyenne une soixantaine d'heures par semaine, la révélation de tels salaires a tout pour faire fuir la majorité des jeunes les plus motivés qui avait, l'espace d'un instant, esquissé l'idée de devenir médecin.

La stigmatisation dont sont victimes les médecins de la part de certains politiques et certains médias ne va pas seulement décourager les jeunes d'embrasser cette profession mais a déjà des effets pervers sur la relation médecin-malade et en particulier sur la confiance du patient. L'une des conséquences, que l'on observe aujourd'hui au cabinet médical, est de pousser le patient à demander un deuxième, voire un troisième avis et ainsi d'augmenter les coûts!

\section{Lorsque l'on sait qu'il faut une douzaine d'années pour former un médecin et d'avantage pour un chirurgien, la revalo- risation de leur travail est une urgence et une priorité}

Les responsabilités de l'Etat dans cette gestion déplorable du système de santé et dans la désinformation du public sont accablantes, comme le seront probablement les conséquences des décisions prises sur des bases erronées.

Les responsabilités de l'Etat dans cette gestion déplorable du système de santé et dans la désinformation du public sont accablantes,

Il est temps pour nos dirigeants de s'entourer de personnes compétentes, de réfléchir l'espace d'un instant, de cesser de trouver des boucs émissaires et de se mettre au travail, c'est le fond qui manque le moins [7].

Collusion d'intérêts

Absence de collusion d'intérêts

\section{Références}

1 Molière. Le Malade imaginaire, Bibliothèque de la Pléiade, Scène IX, page 1496. Paris: Editions Gallimard; 1971.

2 Hugon O. Santé: la grippe coûte 200 millions par année à l'économie et 100 millions en frais médicaux. Le Nouvelliste, 23 novembre 2017. Repris de la RTS, documentaire du 23 janvier 2017 citant l'Office fédéral de la santé publique (OFSP).

3 Alt JL. Porte-parole de la SUVA dans le TTC de la RTS du 12 janvier 2015 et courriel du 29 mars 2018.

4 Schlup J. La médecine ambulatoire de demain, victime du financement d'hier. Bulletin des médecins suisses. 2017;98:25.

5 Indécent, dans le sens de ce qui choque par sa démesure.

6 Eggimann P, Repond PA. Communiqué de presse de la SVMRéaction aux propos d'Alain Berset au TJ de la RTS du 29 janvier 2018

7 La Fontaine J. Fables, livre cinquième, Fable IX, Le laboureur et ses enfants, page 141. Paris: Editions Garnier Frères; 1962. 